\title{
Scattered Data Interpolation: Tests of Some Methods*
}

\author{
By Richard Franke
}

\begin{abstract}
This paper is concerned with the evaluation of methods for scattered data interpolation and some of the results of the tests when applied to a number of methods. The process involves evaluation of the methods in terms of timing, storage, accuracy, visual pleasantness of the surface, and ease of implementation. To indicate the flavor of the type of results obtained, we give a summary table and representative perspective plots of several surfaces.
\end{abstract}

1.0. Introduction. The basic problem which is being addressed here is evaluation of methods for obtaining a smooth (at least continuous first partial derivatives) bivariate function, $F(x, y)$, which takes on certain prescribed values, $F\left(x_{k}, y_{k}\right)=f_{k}$, $k=1, \ldots, N$. The points $\left(x_{k}, y_{k}\right)$ are not assumed to satisfy any particular conditions as to spacing or density, hence the term "scattered." It is usually convenient to think of the values $f_{k}$ as arising from some underlying (not necessarily known) function $f(x, y)$, so that $f_{k}=f\left(x_{k}, y_{k}\right), k=1, \ldots, N$.

The problem of interpolation of scattered data in two or more independent variables has been addressed by numerous authors, as can be seen by the bibliography. Many of the basic ideas involved are discussed in two survey papers (both over a wider class of approximations than we consider here) due to Schumaker [52] and Barnhill [4]. A recent review of methods for contouring, which treats many of the same ideas from that point of view, is given by Sabin [51]. Many ideas put forth have not previously been explored computationally, or only to a limited extent. Thus, the capabilities of some plausible ideas were unexplored. In addition, most of the methods involve one or more ad hoc assumptions requiring a user to specify parameters (one or more). Generally only cursory attention has been paid to the appropriate choice of these parameters, and their overall effect on the interpolant has usually not been determined.

Out of this situation arose a desire to attempt to answer a number of questions, basically all related: Which of these many methods deserve further study and development, and which should be discarded? Some methods require the user to specify an ad hoc parameter, and we have investigated the possibility of using a standard default value. The default value should give reasonably good results over a number of different sets of data, and preferably the interpolant should be rather stable with respect to changes in the parameter. Additionally, it is convenient for the user if the parameter is related to something about the data which can be easily

Received July 21, 1980; revised May 6, 1981.

1980 Mathematics Subject Classification. Primary 65D05; Secondary 65D15.

* Supported in part by the Foundation Research Program at the Naval Postgraduate School. 
estimated. In many cases (perhaps all), subjective judgements must be made about these matters, although some firm information can be obtained.

Some previous fairly extensive work had been done by McLain [41] which inspired a somewhat similar study of another class of ideas by the current investigator [16]. The initial thrust of the investigation was to compare a few "local" methods to determine which seem to work reasonably well. As the investigation proceeded, more ideas were supplied by colleagues and others so that in the end, more than a few methods are tested and compared here, including "global" methods. The total number of programs involved in this study is 32 , some of which are fairly minor variations of others.

The concept of a "global" method is easily understood. The interpolant is dependent on all data points, and addition or deletion of a data point, or a change of one of the coordinates of a data point, will propagate throughout the domain of definition. The idea of a "local" method is not so clear. Typically one thinks of it as meaning that addition or deletion of a point, or a change of one of the coordinates of a data point, will affect the interpolant only at nearby points, that is, the interpolant will be unchanged at distances greater than some given distance. There are some difficulties here. If the data (the $\left(x_{k}, y_{k}\right)$ points) are "random", one must inspect (in some way) all the data to determine which are "nearby". Does this mean there is no such thing as a "local" method? (Rosemary Chang first mentioned this idea.) We have taken a somewhat more liberal view of "local" and take it to mean that the interpolant involves only "nearby" points and one or more parameters. We allow the parameters to have been globally determined as a matter of user convenience, even though a (successful) argument can be made that then the method is not local. Thus, we classify methods as local or global without regard to how parameters are chosen or computed.

The use of global methods is not feasible for very large $N$ since they of ten involve the solution of a system of $O(N)$ equations (often exactly $N$ ) and in any case involve processing all points. When systems of equations must be solved, the systems are often full and not necessarily well conditioned. While our primary aim was to investigate local methods suitable for very large data sets (several hundred points up to some millions, say), in many instances local methods involve the use of global methods on smaller sets which are then "blended" together to obtain a locally defined global interpolant. Thus it makes sense to to test global methods on moderately sized sets of data. By the same token, it is not necessary to test local methods on sets of 10,000 points (say) by virtue of the fact that they are local. If very large sets of data were to be considered, it is clear that a different implementation approach might be necessary, one which would involve a larger amount of preprocessing and perhaps additional storage.

This paper is essentially a condensed version of technical report [18]. The full documentation consists of some 370 pages, nearly 300 pages being devoted to comparative tables and perspective plots obtained by applying 29 algorithms for solution of the scattered data interpolation problem. Each of the methods is described there in some detail along with discussions of its performance in the tests.

1.1. Tested Characteristics of Methods. The characteristics on which various methods are to be compared, and how they are to be weighted in the final analysis, 
are somewhat subjective. While no representation is made that the list is exhaustive (or even close to it), nor that everyone will be in agreement on it, the following items are the ones considered here. We give them and discuss them in order of decreasing importance. In the presentation of information in the summary (tables and perspective plots) each reader may weigh various aspects to suit his own needs.

Accuracy. Accuracy in reproducing a known surface is certainly one important aspect of comparison. In the usual application no representation of the underlying surface $z=f(x, y)$ is known; however, if the method approximates a variety of surface behavior faithfully, we expect it to give reasonable results in other instances. Numbers can be put on the performance of a method tested in this fashion, and we have used this idea extensively.

Visual Aspects. It has developed during the course of this project that the appearance of the interpolant is very important. The most useful representation of the surface is a dynamic one, where different viewing angles can easily be obtained. This could be achieved by building models, as well. Neither of these capabilities is available to the author, and in any case, wide distribution of such representations is impossible. Perspective plots of 3-dimensional surfaces were available and have been used extensively. The resolution and viewpoint of a perspective plot could obscure the fact that a surface is bad, but it is doubtful any truly bad surface has escaped detection.

Visual ratings are often closely related to the accuracy with which an interpolant reproduces test surfaces. There seems to be a closer relationship when accuracy is high since there is less chance for the interpolant to misbehave. At moderate accuracies one interpolant may be visually pleasing while another with similar accuracy is not.

The visual aspect is quite subjective, and ratings by different persons will give somewhat different results, although probably not contradictory ones. While it is felt that the visual aspect is quite important, exactly how this information is integrated into the overall assessment of a method is also a subjective matter.

Sensitivity to Parameters. Many of the tested methods involve the choice of one or more parameters. These choices have generally been converted to ones which are related to mean distances to nearest neighbor, although precisely that idea is never directly used. Here we are talking of nearest neighbor in the set of points $\left\{\left(x_{k}, y_{k}\right)\right\}$. Sometimes the parameter takes the form of an anticipated number of points in the region which defines a local interpolant.

Methods which involve parameters underwent informal testing for suitable values of the parameters. For fixed sets of data, the parameter was varied to find a suitable range for its value. Some methods were quite sensitive to the parameter value. Some methods were apparently sensitive to the dependent-variable values, as well as the $\left(x_{k}, y_{k}\right)$ values. Thus, a parameter value giving good results for one function might yield poor results for a different function sampled at the same points. It is desirable that a method be stable with respect to perturbations in the parameter and that its value not be highly dependent on the function sampled. Such methods were found.

Timing. The computational effort required is generally not of great interest, unless it is very high. In this respect, only one of the methods tested was downrated 
for this reason. Some methods are quite efficient in terms of time required for the calculations. These methods have generally been found deficient in other categories, unfortunately. For methods which involve a preprocessing phase, distinct from an evaluation (of the interpolant) phase, the two times for standard problems are given separately. Execution times were taken from the multiprogramming environment on the IBM $360 / 67$ and as such may vary $10-20 \%$ with exactly the same data. Thus, execution times must be viewed as a guide rather than as precise measurements.

Storage Requirements. As with computational effort, storage requirements are not crucial, unless they are very high. For very large problems this may be altered, of course. We count storage requirements only in terms of additional arrays needed to store data beyond the $\left(x_{k}, y_{k}, f_{k}\right)$ points. No account is taken of simple variables or program length.

Ease of Implementation. Ease of implementation is of no great concern if one obtains a working program. In other instances it may be of considerable importance. The judgement is again subjective. Further, it could be different depending on the philosophy behind the implementation. The form of the implementation could involve trade-offs between timing and storage and would doubtlessly alter the ease of implementation.

Implementation of programs specifically for this project generally was done with a lack of frills. Reasonable care was taken to assure that a grossly inefficient algorithm was not coded, but no doubt it is possible to improve on most of them. In particular, use of some preprocessing and additional storage was not used to increase efficiency during the evaluation phase. For a general purpose program this should probably be done. Some of the documented programs did use these devices. Ease of implementation is generally meant to take into account the complexity of the ideas involved in the method and the amount of code required.

1.2. The Testing Process. The initial tests performed on a few methods eventually gave rise to a standard set of test problems and a set of supporting subprograms to generate statistics from the tests and generate perspective plots of surfaces. Due to the evolution of ideas as the study progressed, some aspects of the process are not as simple as they might have been. This is particularly true of some of the test functions, but this has no bearing on the validity of the tests.

To enable testing many different methods in a consistent manner, and with a minimum of effort, a set of standard subprograms was developed which generate the test cases, compute deviation statistics for known test surfaces, obtain timing statistics, and generate and label perspective plots of the surfaces. With the current set of supporting subprograms it is generally quite easy to test a new method which is typically supplied as a subprogram (or several) which generates the values of the interpolant on a grid of $x-y$ points. Typically all that is required is to set certain parameters, reserve any required workspace, and call the subroutine, all of which can be done with a few statements added to the prototype driver program.

There were six different test functions selected. These exhibit a variety of behavior, and, when sampled over three different $x-y$ data sets of 100,33 , and 25 points, gave a total of 18 data sets. In addition to these, two sets of data were obtained from the literature (from [2] and [13]). One of these [13] was scaled in one 
variable, which revealed something of the effects of scaling variables differently. A fourth $x-y-z$ data set was a cardinal function, giving a total of 22 different data sets. Not all methods were tested on all sets of data; only those readily available methods, or those which performed well in initial test, have complete test results reported.

2.0. Descriptions of Tested Methods and Some Results. For description purposes the methods are classed into six groups: (1) Inverse distance weighted methods, (2) Rectangle based blending methods, (3) Triangle based blending methods, (4) Finite element based methods, (5) Foley's methods and (6) Nodal basis function methods. While there is necessarily a blurring of distinctions across these group lines, they constitute fairly distinct ideas and it is convenient to group them this way. In addition to methods which fall into those groups, a variation of Maude's method [40] has been tested since [18] appeared. While it is somewhat similar to methods of group (1), and while Maude's method also led to the methods of group (2), it will be discussed separately as group (7) Modified Maude methods.

2.1. Inverse Distance Weighted Methods. The original inverse distance weighted interpolation method is due to Shepard [53]. All methods of this type which we consider may be viewed as generalizations of Shepard's method, or variations of such generalizations. The basic Shepard's method is

$$
F(x, y)=\sum_{k=1}^{N} w_{k}(x, y) f_{k} / \sum_{k=1}^{N} w_{k}(x, y),
$$

where $w_{k}(x, y)=d_{k}^{\mu}$, and typically $\mu=2$, although other values may be used. Here $d_{k}=\left(\left(x-x_{k}\right)^{2}+\left(y-y_{k}\right)^{2}\right)^{1 / 2} \cdot \mu$ may be replaced by $\mu_{k}$ and could possibly be different for each $k$. Several authors have considered various aspects of Shepard's method [4], [5], [21], [52].

Shepard's method is a global method, and the original paper suggested a scheme for localizing it by piecing together a parabolic segment with $d_{k}^{-2}$ in such a way as to obtain a $w_{k}$ which is zero outside some disk, say of given radius $R$, centered at $\left(x_{k}, y_{k}\right)$, and which is still $C^{1}$. A simpler and more natural scheme suggested by Franke and Little [4, p. 112] is used in much of this work, that is,

$$
w_{k}(x, y)=\left[\frac{\left(R-d_{k}\right)_{+}}{R d_{k}}\right]^{2} .
$$

Shepard's method has an undesirable property for general use in that a flat spot occurs at each data point. Use of information about derivatives, either given or generated from the data, was suggested by Shepard and resulted in an approximation of the form

$$
\begin{aligned}
& F(x, y) \\
& \quad=\sum_{k=1}^{N} w_{k}(x, y)\left[f_{k}+\left(\frac{\partial f}{\partial x}\right)_{k}\left(x-x_{k}\right)+\left(\frac{\partial f}{\partial y}\right)_{k}\left(y-y_{k}\right)\right] / \sum_{k=1}^{N} w_{k}(x, y) .
\end{aligned}
$$

More generally, one may consider approximations of the form

$$
F(x, y)=\sum_{k=1}^{N} w_{k}(x, y) L_{k} f(x, y) / \sum_{k=1}^{N} w_{k}(x, y),
$$


where $L_{k} f$ is an approximation to $f$ such that $L_{k} f\left(x_{k}, y_{k}\right)=f_{k}$. This is the basis for several of our methods. In this context we refer to the $L_{k} f$ as nodal functions.

Another way in which Shepard's method can be generalized is to view the method as an inverse distance weighted least squares approximation to $f(x, y)$ by a constant. One can then generalize to an approximation taking the form

$$
F(x, y)=\tilde{F}\left(a_{0}, a_{1}, \ldots, a_{n} ; x, y\right),
$$

where $a_{0}, \ldots, a_{n}$ are parameters chosen by taking them to minimize (for a given $(x, y))$ the expression

$$
\sum_{k=1}^{N}\left[f_{k}-\tilde{F}\left(a_{0}, a_{1}, \ldots, a_{n} ; x_{k}, y_{k}\right)\right]^{2} w_{k}(x, y)
$$

This approach was taken by McLain [41] in evaluating a number of methods where $\tilde{F}$ was taken as a linear combination of low order monomials and $w_{k}(x, y)$ as $d_{k}^{-2}$ or $\exp \left(-\alpha d_{k}^{2}\right) d_{k}^{-2}$. McLain also considered some approximations where $f$ entered nonlinearly. We have considered one of McLain's methods and a variation of another. All of the methods of this class may be derived as variations of the above formula for $\tilde{F}$ [19].

Some papers discussing theoretical aspects of the above generalizations of Shepard's method have appeared recently [34], [33]. During revision of this paper, the details of two papers came to the attention of the author. Each gives, at an earlier publication date, a method previously attributed to others. Crain and Bhattacharyya [8] give the simplest version of Shepard's method, while Pelto, et al. [48], give the inverse distance weighted quadratic method credited to McLain.

The performance of methods in this group is very dependent on an appropriate weight function, $w_{k}(x, y)$ in (4) or (6). $w_{k}=d_{k}^{-2}$ is unacceptable since it allows too much influence by far away points, even when, for example, the $L_{k} f(x, y)$ in (4) are reasonably good local approximations. The use of polynomials of degree $<2$ for the $L_{k} f(x, y)$ is inadequate to describe the local behavior of the surface. McLain's quadratic version of (6), with $w_{k}=\exp \left(-\alpha d_{k}^{2}\right) d_{k}^{-2}$, performs well, but is extremely time consuming. Best performance in the group is achieved by a version of (4) using quadratic approximations for the $L_{k} f$ and $w_{k}$, given by (2), for an appropriate $R$. We have called this the Modified Quadratic Shepard's Method. It is developed from (6) in [19], and pertinent theoretical results are given in [34].

2.2. Rectangle Based Blending Methods. The basis for this class of methods is discussed in [16] and was inspired by a short paper by Maude [40] which generalized the idea of deficient quintic splines to several variables. Unfortunately, the original interpolation function exhibits rather poor behavior and has not even been included in our tests. The original idea was to represent the interpolation function as

$$
F(x, y)=\sum_{k=1}^{N} w_{k}(x, y) Q_{k}(x, y) / \sum_{k=1}^{N} w_{k}(x, y),
$$


where $Q_{k}(x, y)$ is the quadratic polynomial interpolating $f(x, y)$ at $\left(x_{k}, y_{k}\right)$ and the five nearest neighbors to $\left(x_{k}, y_{k}\right)$ from the set $\left\{\left(x_{j}, y_{j}\right)\right\}$, and

$$
w_{k}(x, y)= \begin{cases}1-\left(\frac{d_{k}}{R_{k}}\right)^{2}\left(3-2 \frac{d_{k}}{R_{k}}\right), & d_{k} \leqslant R_{k}, \\ 0, & d_{k}>R_{k},\end{cases}
$$

where $R_{k}$ is the distance between $\left(x_{k}, y_{k}\right)$ and its fifth closest neighbor. This idea was generalized to include any $w_{k}(x, y)$ which have finite support (to make the method local) so long as the $Q_{k}(x, y)$ interpolate $f(x, y)$ at all $\left(x_{j}, y_{j}\right)$ where $w_{k}\left(x_{j}, y_{j}\right) \neq 0$. Use of approximations $Q_{k}(x, y)$ in Hilbert spaces, particularly in Sard spaces, was suggested and implemented [17]. One of the chief advantages of this approach is that instead of taking $w_{k}$ with disks centered at the $\left(x_{k}, y_{k}\right)$ as support regions, it is easy to use a smaller number of overlapping rectangles in such a fashion that at most four terms in the sum are nonzero, and $w_{k}(x, y) \equiv 1$. Use of rectangles also simplifies the problem of determining which terms are nonzero and thus results in a faster algorithm.

The set of rectangles is chosen to attempt to make each rectangle contain a given fixed number of points. Suppose the rectangles are defined by grid lines at $x=\tilde{x}_{0}$, $\tilde{x}_{1}, \ldots, \tilde{x}_{n_{x}+1}$ and $y=\tilde{y}_{0}, \tilde{y}_{1}, \ldots, \tilde{y}_{n_{y}+1}$. Then weight functions with support $\left[\tilde{x}_{i-1}, \tilde{x}_{i+1}\right] \times\left[\tilde{y}_{j-1}, \tilde{y}_{j+1}\right]=R_{i j}$ are formed from piecewise Hermite polynomials, local interpolation functions $Q_{i j}$ are constructed so that $Q_{i j}\left(x_{k}, y_{k}\right)=f_{k}$ whenever $\left(x_{k}, y_{k}\right) \in R_{i j}$, and then the overall approximation takes the form

$$
F(x, y)=\sum_{i, j} w_{i j}(x, y) Q_{i j}(x, y)
$$

Any type of local interpolation function $Q_{i j}$ could be used. The author previously suggested Sard type approximations [17]. These have some undesirable properties in that they depend on factors other than relative position of $\left(x_{k}, y_{k}\right)$ points. A second implementation using "thin plate splines" (see Section 2.6) was also tested. Neither of the methods performs as well as the author expected. It would seem that the method should be nearly as good as the underlying local approximation, however, this was not quite borne out by the tests, although the version using "thin plate splines" performs well.

Recently, some work due to Jancaitus, Junkins, and coworkers [30]-[32] has come to the investigator's attention. This work involves the idea of weighted local approximations in a similar fashion and was applied to the problem of terrain modeling. In their case the local interpolation functions were replaced by least squares approximations by polynomials and thus interpolation was not achieved.

2.3. Triangle Based Blending Methods. These methods are conceptually the same as those given by Eq. (4), but a significant difference is that the weight functions are based on a triangulation of the convex hull of the point set $\left\{\left(x_{k}, y_{k}\right)\right\}$. Several such schemes have been proposed, e.g., [7], [19], [20], and [42]. One of those considered here is the one described in [19].

Assume a triangulation of the convex hull, and suppose $(x, y) \in \bar{T}_{i j k}$, where $\bar{T}_{i j k}$ is the triangle with vertices $\left(x_{i}, y_{i}\right),\left(x_{j}, y_{j}\right)$, and $\left(x_{k}, y_{k}\right)$. We then take

$$
F(x, y)=w_{i}(x, y) Q_{i}(x, y)+w_{j}(x, y) Q_{j}(x, y)+w_{k}(x, y) Q_{k}(x, y),
$$


where the weight functions are finite element "shape" functions satisfying $w_{m}\left(x_{n}, y_{n}\right)=\delta_{m n}$ and the nodal functions $Q_{n}$ satisfy $Q_{n}\left(x_{n}, y_{n}\right)=f_{n}$ for $m, n=$ $i, j, k$. In all previously referenced methods the weight functions may be viewed as nine-parameter cubic shape functions with a rational correction to obtain normal derivatives equal to zero, and hence a $C^{1}$ approximation overall. There are many ways to obtain such correction terms, all of which appear to lead to the possibility of negative values being taken on by one of the weight functions if the triangle is very obtuse. This is probably not serious, although one has no control over the shape of the triangle in the sense that very obtuse angles cannot be avoided, especially near the boundary of the convex hull. The weight functions used here are obtained from a minimum norm problem [45]. Let $b_{i}, b_{j}, b_{k}$ be the barycentric coordinates of $(x, y)$ in $\bar{T}_{i j k}$, and let $l_{i}, l_{j}, l_{k}$ be the lengths of the sides opposite vertices $i, j$, and $k$, respectively. Then the weight function is given by

$$
w_{k}(x, y)=b_{k}^{2}\left(3-2 b_{k}\right)+6 b_{i} b_{j} b_{k}\left[\alpha_{k j}+\alpha_{k i}\right] \text {, }
$$

with

$$
\alpha_{k j}=\frac{b_{k} b_{j}\left(1+b_{i}\right)}{\left(1-b_{i}\right)\left(1-b_{k}\right)}\left[\frac{l_{k}^{2}+l_{i}^{2}-l_{j}^{2}}{2 l_{i}^{2}}\right],
$$

and the others are obtained by a cyclic permutation of the indices.

While the basic method is defined only on the convex hull of the point set, it is easily extended to a globally defined function by the following idea. The exterior of the convex hull is divided into semi-infinite rectangles and semi-infinite triangles by constructing perpendiculars to the exterior edges of the convex hull at each exterior vertex. The value of the interpolant at an exterior point is obtained from the nodal function values at one (triangular area) or two (rectangular area) nearest points.

The $Q_{n}$ in (9) can be taken to be any function having the required property. As with the inverse distance weighted methods, linear functions are inadequate. Use of appropriate quadratic functions yields results similar to those obtained from (4) in that case. Certain advantages accrue here. The evaluation phase is very fast since only three terms appear in (9), and the algorithm for determination of which triangle a point lies in is fast. Disadvantages are that a large amount of auxiliary storage is required for the triangulation (incidentally the triangulation algorithm itself is very fast), and long slim triangles sometimes yield surfaces which appear to have discontinuities along these triangles because of very rapid changes in function value across the narrow part.

2.4. Finite Element Based Methods. These methods are based on the concept of using $C^{1}$ finite element functions on a triangulation of the convex hull of the point set. This requires a scheme for estimating some derivatives (which derivatives depends on the element used by the method) at the data points. Our test results indicate that accurate estimates of the derivatives are very important and have a pronounced effect on the visual aspects of the surface as well as the accuracy. Three methods of this type, each using a different element, were tested. One was tested with several variations in the way partial derivatives are estimated. An additional scheme has been tested since [18] appeared, and we mention it here as well. 
Akima's method [2], [3] is readily available. It uses the $C^{1} 18$ parameter quintic finite element. Extrapolation outside the convex hull is provided. The element requires estimates of first and second partial derivatives at the data points. In standard form a certain average of slopes of planes through the data point and each pair of several nearest neighbors is used to determine first derivative estimates. Second derivatives are estimated by applying the process to the derived data. Two variations of this scheme (by varying the weights in the average) were tested, as well as a version which obtained the derivatives from a local quadratic approximation. Performance of the method depends greatly on the estimates of the derivatives. The latter version gives the best results but at a considerable time penalty in the preprocessing phase. The published version is by far the fastest algorithm tested here, but gives poor results in some instances due to poor derivative estimates, generally, and sometimes due to long slim triangles in the triangulation. The latter is unavoidable in triangle based methods and often occurs. It cannot be avoided without abandoning the convex hull, or adding fictitious points.

Since the appearance of [18], Akima has proposed a variation in the computation of derivatives. Instead of using nearest neighbors in the usual sense, the neighbors in the triangulation are used. This scheme generally gives poorer surfaces than the original method, especially near the boundaries of the convex hull, where extraneous bumps often occur. This version is available in edition 8 of the IMSL library as subroutine IQHSCV.

Lawson's method [35] is similar in spirit to Akima's except that the CloughTocher element is used. First partial derivatives are required, and these are obtained from a quadratic approximation. Results are generally better than for Akima's method, although execution times are greater. Lawson's program does not extrapolate outside the convex hull.

Nielson's minimum norm network [46] uses a cubic element with a rational correction to achieve a $C^{1}$ function. The element is the solution of a certain minimum norm problem [45] and requires first partial derivatives in its discretized form. These are obtained by assuming a cubic variation along each edge in the triangulation and minimizing the integral (over all edges in the triangulation) of the second derivative squared. This gives the best results in this class of methods. It is somewhat slower than the other methods, but could probably be improved considerably in the evaluation phase. The method does not provide extrapolation outside the convex hull, although the investigator provided $C^{0}$ extrapolation for the tested version. Nielson's method is global as opposed to Akima's and Lawson's, which are local. The system of equations for the partial derivatives is solved by an iterative process which converges rapidly.

Since the appearance of [18], Little's method [36] has been tested and performed very well. It is based on the use of a cubic element with a rational correction term. Partial derivatives are estimated using a weighted average of the slopes of planes through neighboring points in the triangulation. One significant difference from other schemes in this group usually results in better control over long slim triangles. That difference is abandonment of the convex hull by extrapolating for a function value at some added exterior points. These points are then added to the set, which is retriangulated. This eliminates the usual edge effect, but, depending on the 
extrapolated function value, can distort the surface near the edge if it is not representative of its behavior near the boundary of the convex hull.

Other finite elements could be used. One which might be appropriate is the piecewise quadratic due to Powell and Sabin [49]. This element was designed for contouring, hence the desirability of a quadratic. For general application the large number of subtriangles involved would seem to be a detriment. The author has not had access to a program based on this scheme, but it is likely it would perform on about a par with others considered here.

2.5. Foley's Methods. Foley's methods [14], [15] involve several ideas. The use of a generalized Newton type interpolant is involved in them prominently. Another idea which is exploited successfully is that of using one interpolant to generate a grid of points on which product type approximations can be constructed. The product approximation will not, in general, interpolate the given data. Hence a correction based on the original approximation is made to the error. This process is termed a "delta sum" by Foley, written $P \Delta Q$, defined by $P \Delta Q=P \oplus Q P$, and implemented as $(P \Delta Q) f=P(I-Q P) f+Q P f$.

The idea has greater generality than considered by Foley, but the application of it seems to be the appropriate one. He considers cases where the product type approximation (taking the part of $Q$ ) is either the bivariate product Bernstein polynomial or the bivariate product natural bicubic spline. The first interpolant (taking the part of $P$ ) is taken as either the generalized Newton interpolant, or a form of Shepard's method. The delta sum idea is applied in iterated form for two methods.

The generalized Newton interpolant takes the form

$$
T_{N}(x, y)=\sum_{k=1}^{N} a_{k} w_{k}(x, y), \quad \text { where } a_{k}=\frac{f_{k}-T_{k-1}\left(x_{k}, y_{k}\right)}{w_{k}\left(x_{k}, y_{k}\right)},
$$

and $w_{k}(x, y)$ has the property $w_{k}\left(x_{i}, y_{i}\right)=0, i=1,2, \ldots, k-1$. This function is dependent on the order of the points, and so Foley's scheme involves an ordering process.

The best performance is provided by the iterated delta sum method using the generalized Newton polynomial with natural bicubic splines. The method performs reasonably well, but sometimes exhibits "polynomial-like" ripples in the surface, although it generally gives quite smooth surfaces.

2.6. Global Basis Function Type Methods. These methods can be characterized by the following idea. For each $\left(x_{k}, y_{k}\right)$ simply choose some function $G_{k}(x, y)$, and then determine coefficients $A_{k}$ so that $F(x, y)=\Sigma_{k} A_{k} G_{k}(x, y)$ interpolates the data. Schemes which work are not so simple in that appropriate choices of functions $G_{k}$ are not particularly easy to make. Even if the functions $G_{k}$ have only local support, the methods are global and further they require solution of a system of $N$ linear equations. In all instances we consider, the systems have a symmetric coefficient matrix $\left(G_{i}\left(x_{j}, y_{j}\right)\right)$, but this need not be the case. Usually the $G_{k}$ are really functions of the one variable $d_{k}$. Numerous colleagues have suggested (among others) $B$-splines, Gaussian distributions, and other basis functions which seem to have an at best shaky mathematical justification. These schemes involve parameters to be specified by the user. For a Gaussian distribution function it is 
the variance, while for rotated $B$-splines it is the radius at which the function becomes zero. These two methods are quite sensitive to the parameter, and, while good results are possible, the appropriate value of the parameter seems to depend on the function value as well as the $\left(x_{k}, y_{k}\right)$ points, which is an undesirable characteristic. A potentially undesirable feature of many of these schemes is that they usually have no polynomial precision, e.g., not even constant functions are reproduced exactly. Based on practical experience, however, it is this author's opinion that incorporating polynomial precision does not, in itself, yield significant improvement. This observation has also been made elsewhere [14].

In terms of fitting ability and visual smoothness, the most impressive method included in the tests is the "multiquadric" method, due to Hardy [23]-[29]. In this method the $G_{k}$ 's are taken to be the upper sheet of a hyperboloid of revolution, $G_{k}=\left(d_{k}^{2}+r^{2}\right)^{1 / 2}$. Here $r$ is a parameter to be specified by the user. The method is quite stable with respect to this parameter and yields consistently good results, of ten giving the most accurate results of all tested methods. The surfaces are usually pleasing and very smooth. Results nearly as good are obtained with the "reciprocal multiquadric" method, $G_{k}=\left(d_{k}^{2}+r^{2}\right)^{-1 / 2}$. However, here the choice of $r$ is somewhat more crucial since small values of $r$ will lead to a surface of peaks and dips at each data point.

Two methods which have basis functions similar to the multiquadric method are due to Duchon [9]-[12] and are also treated by Meinguet [43]-[44]. Unlike Hardy's, which as yet has no theoretical basis, these methods have an elegant theory in a Hilbert space setting. In one case $G_{k}=d_{k}^{3}$, while in the other $G_{k}=d_{k}^{2} \log d_{k}$. The latter minimizes the thin plate functional

$$
\int_{R^{2}}\left(\left|\frac{\partial^{2} f}{\partial x^{2}}\right|^{2}+2\left|\frac{\partial^{2} f}{\partial x \partial y}\right|^{2}+\left|\frac{\partial^{2} f}{\partial y^{2}}\right|^{2}\right) d x d y
$$

in a certain Hilbert space and is termed a "thin plate spline". In each case the approximation contains a linear combination of functions in the kernel of the functional (that is, a linear function), along with side conditions, the geometric effect being to remove terms which grow faster than linear as one moves far away from the data. The thin plate splines had previously been discovered by Harder and Desmarais [22], where they are called surface splines. The two methods generally perform in comparable fashion, but the thin plate spline leads to coefficient matrices with smaller condition numbers, and hence was the more extensively tested of the two. The thin plate splines generally give approximations nearly as good as the multiquadric method, pleasant visually, and very smooth. This method has no parameter and, like other methods tested in this class, has the desirable properties of translation and rotation invariance.

It would seem that functions $G_{k}$ which diminish as one moves away from the point $\left(x_{k}, y_{k}\right)$ would yield better results than the ones which increase with distance. The reasons for thinking this is that a large value far away means a basis function has more influence far away than at the point with which it is associated. Also, the coefficient matrix for the system giving the weights is full, with its largest elements off the diagonal. Nonetheless, methods which performed the best have basis functions which are unbounded. 


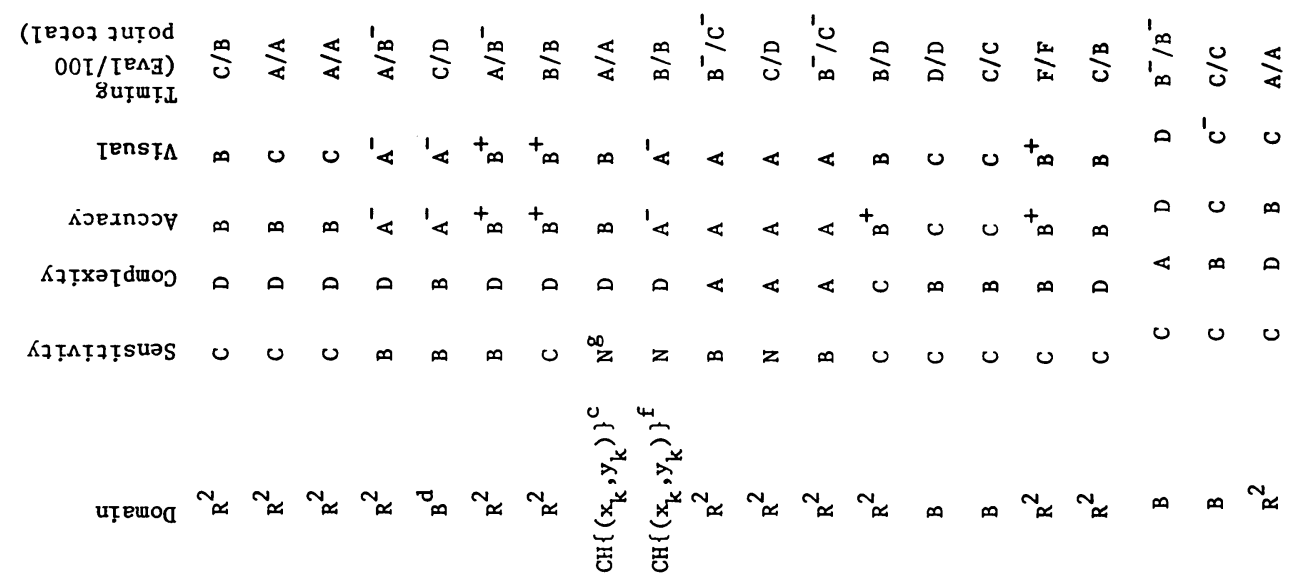




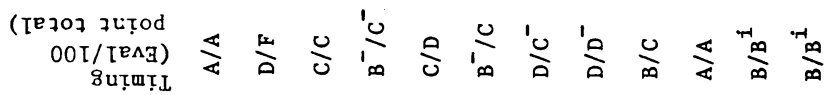

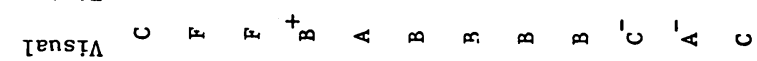

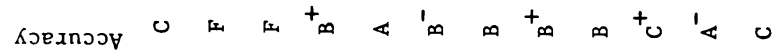

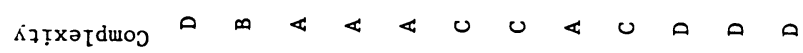

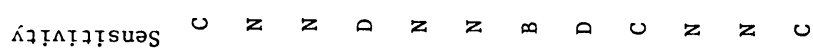

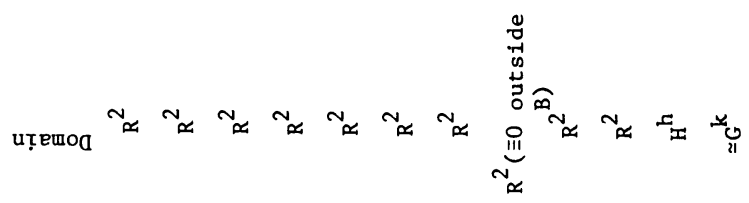

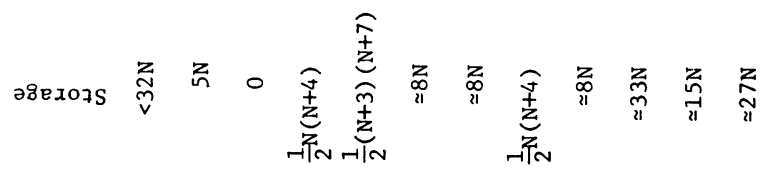

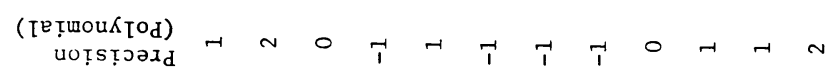

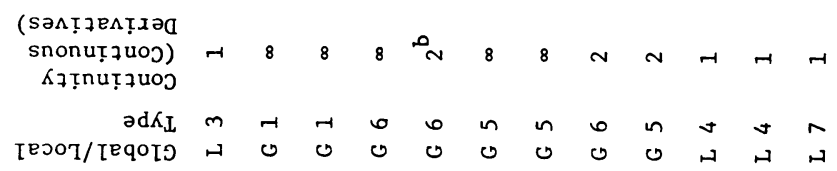

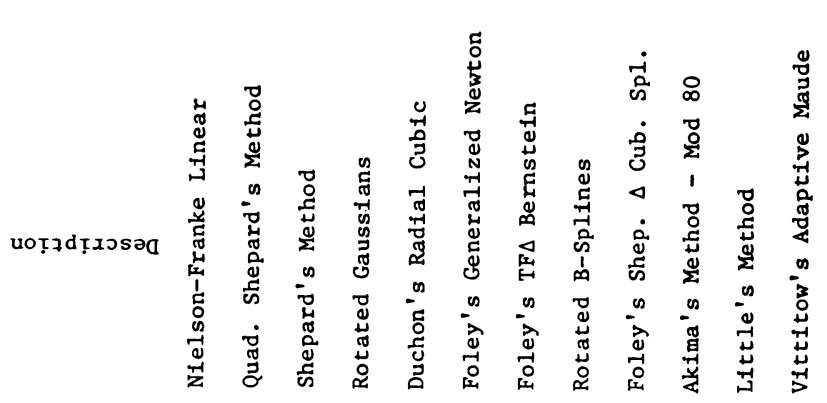

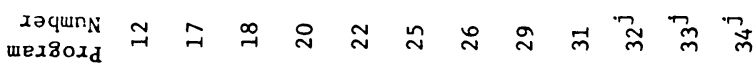

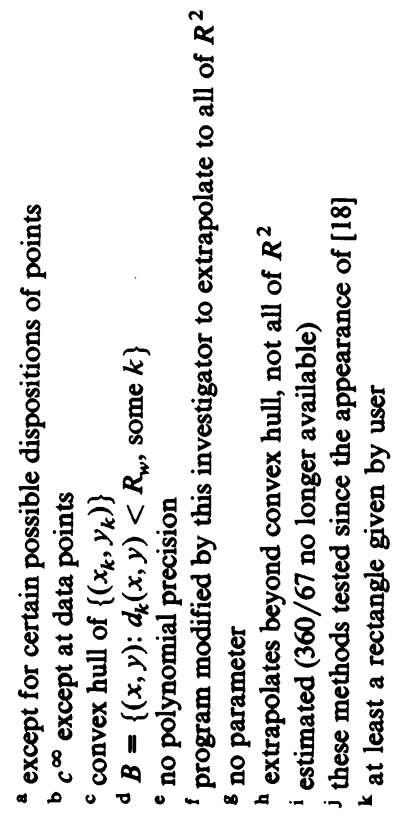


We have not directly tested any method based on the idea of "kriging", or "regionalized variables", due to Matheron [38], [39] and discussed by numerous others, e.g., [47], [1], [37], [50]. However, it appears that kriging methods are related to global basis function methods, and indeed are identical to them under certain conditions. The statistical assumptions and approach taken in Kriging make the method appear harder, computationally, although this viewpoint allows estimation of the goodness of fit. The assumptions made seem to this author to be related to choice of a good parameter value in global basis function methods.

2.7. Modified Maude Methods. We briefly discussed Maude's method [40] in Section 2.2 and noted that it did not perform very well. This is primarily due to poor behavior of interpolating quadratics in two variables. Vittitow [54] has developed some modifications of the idea which attempt to alleviate this problem, as well as to overcome the possibility of "holes" appearing in the domain due to varying sparseness of the data.

Poor behavior of the local interpolation functions (the $Q_{k}$ 's in Section 2.2, Eq. (7)) is improved by (1) reducing the number of interpolation points, and (2) increasing the total number of points used to define the local interpolation function. This is achieved by calculating a constrained (to interpolation at a reduced number of points) least squares fit to a larger set of nearby points. Quadratic, cubic, or quartic functions can be used.

Complete coverage of a specified domain is achieved by adaptively determining the disks on which $w_{k}(x, y)$ (in Section 2.2, Eq. (7)) is nonzero. In the process, disks are no longer centered at the data points and fewer than $N$ are usually needed. The actual number of interpolation points varies from disk to disk, but is no greater than a specified number. The number of points included in the least squares process (in addition to the interpolation points) is also specified by the user.

3.0. Summary. Numerous tables in [18] summarize the results of the study. In particular, there are tables giving

-maximum, mean, and rms deviations of surfaces generated by data taken from known functions;

-best performance in the accuracy tests among local methods, and overall;

-effect of varying the parameter, if any;

- times for preprocessing, interpolant evaluation, and total time;

-a summary, giving an overall "quick look" at the results.

The summary table is reproduced here as Table 1, including results for the three subsequently tested programs. We briefly describe each column in the table. Footnotes are referenced by small letters. Program number is a number assigned to the program and used to identify it in plots and tables. Description is a brief pointer to the person or ideas involved. Global/Local tells whether the method depends globally on the data (G) or locally (L). Type gives the subsections of article 2 into which the method falls. Continuity indicates the highest order derivatives of the interpolant which are all continuous. Precision refers to the highest degree polynomial which is reproduced exactly by the interpolant. Storage refers to estimated size of storage arrays required in addition to the given data. No account of scalar variables or program size is included. Domain is the domain of definition of the 
interpolant. Sensitivity to parameters is a purely subjective score, based on informal testing of the scheme. Included were whether some value of the parameter worked well for a variety of surfaces for a given set of $(x, y)$ points, and whether the interpolant was stable with respect to changes in the parameter from that value. Complexity simply reflects the investigator's perception as to the complexity of ideas involved and the ease of implementation into a computer program. Accuracy is again subjective and is based on the relative amount of deviation one might expect from the true surface for a given method. Of course, perusal of the deviations tables will reveal that some methods do well on some surfaces and not so well (relatively speaking) on others. Visual pleasantness is a subjective rating based on perspective plots of the interpolant. Timing is relatively well defined. The first letter represents the sum of the evaluation times for three cases of 100,33 , and 25 data points. Ranges for A, B, C, D, and F, respectively, are $(0,7],(7,21]$, $(21,30],(30,50]$, and $(50, \infty)$. The second letter represents the total time for 100 data points and 1089 evaluation points. Ranges are $(0,4],(4,12],(12,20],(20,30]$, and $(30, \infty)$. The first 13 lines in the table give the results for the extensively tested methods. The remaining lines give results for less extensively tested methods and the three subsequently tested methods.

To give the flavor of the type of visual information included in the report, two pages are reproduced here in Figures 1 and 2 . Figure 1 gives the test surface in part (a) and reconstructions of it by the multiquadric method for three different data sets with 100, 33, and 25 points in parts (b), (c), and (d), respectively. Figure 2 shows surfaces generated by the rectangle based blending method due to the author, using thin plate splines as the local approximations. Part (a) is a cardinal function, part (b) was generated from Akima's data, and parts (c) and (d) were generated from Ferguson's data. As a general rule, the best global methods seem to result in surfaces which are visually more pleasant than those obtained from local methods, as though localizing the surface loses something, which, while small, is still significant in that respect. Poor behavior near edges of the data set is more prevalent for local methods. For data sets of up to 100-200 points, global methods are feasible and should be considered. Nielson's minimum norm network can probably be used on somewhat larger sets of data since the sparse system of equations is solved by iteration, while other global methods generally require solution of a full system of $N$ or more equations. Choice of a method for a large number of points is to a certain extent a personal matter, but the previously mentioned Modified Quadratic Shepard's Method performs well, requires moderate storage and computation time, and is relatively easy to implement. It is also easily extended to more independent variables. The triangle based programs, of which Akima's is the most readily available, require considerable machinery and storage for the triangulation, but in the end they are quite fast (Akima's being by far the fastest of all tested methods). These methods are extremely difficult (if not impossible) to extend to more than two independent variables and have other previously mentioned potential defects.

Despite the number of ideas explored and programs written or obtained from authors, and tested, there are still some which were not investigated. In addition to the two methods from the CAGD group at Utah, which were recently obtained, 
there are still more ideas which have arisen there. Many of these are based on triangulations, which the investigator feels are more suited to the design problem (where long slim triangles can be avoided) rather than the interpolation problem. Another idea which was not tested has its genesis in Briggs [6], and is available commercially [55]. The user's manual contains some impressive material, but no tests of the software have been conducted. There are no doubt more ideas worthy of investigation appearing in the literature.

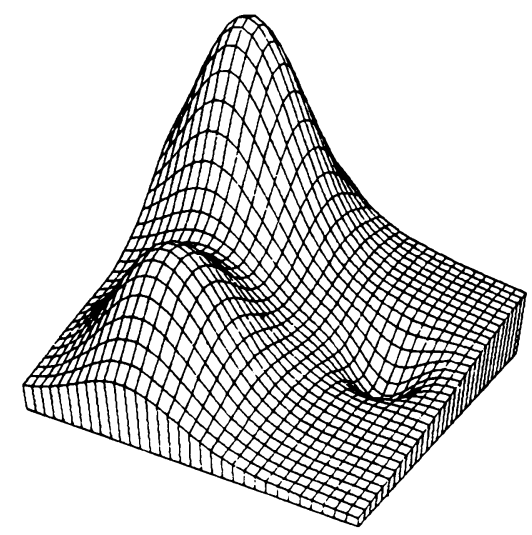

(a) Test surface

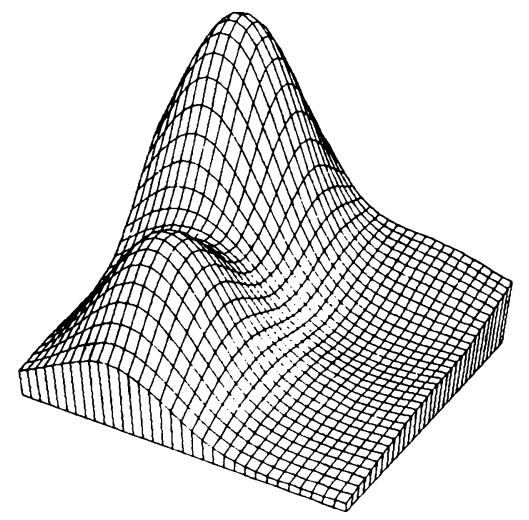

(c) 33 point sample

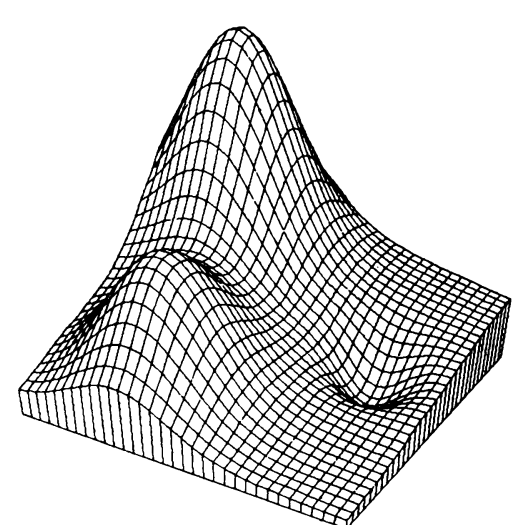

(b) 100 point sample

Figure 1

Hardy's multiquadric method 


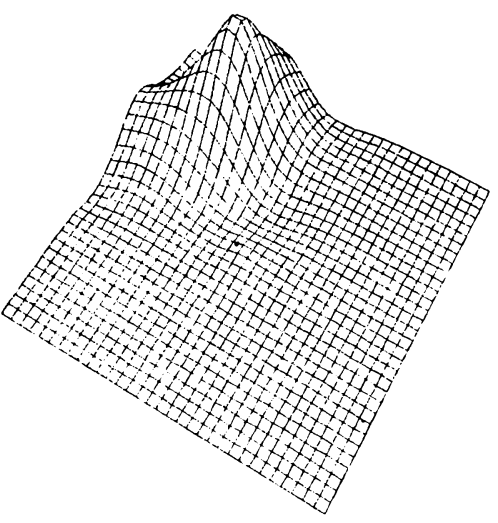

(a) Cardinal function, 2.5 points

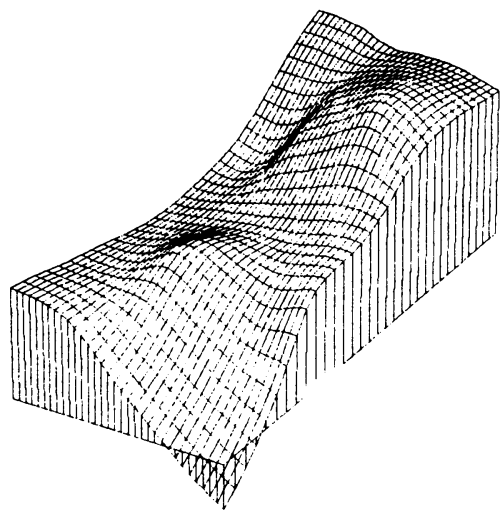

(c) Ferguson's data, 25 points

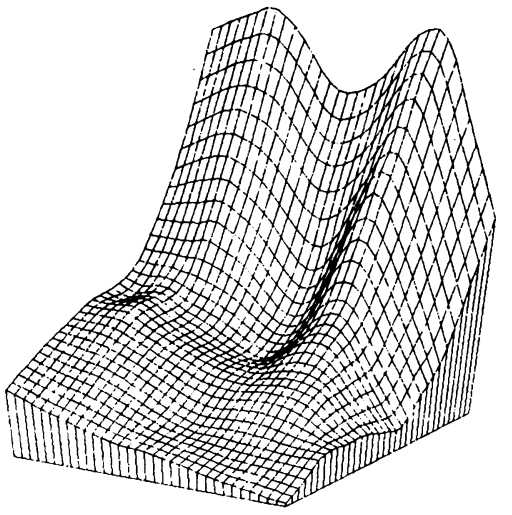

(b) Akima's data, 50 points

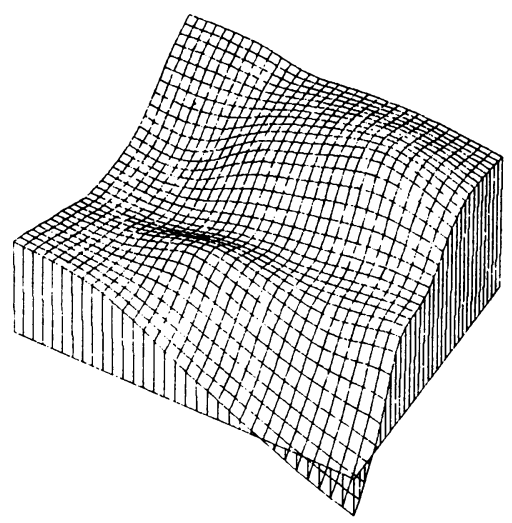

(d) Ferguson's data, yx3, 25 points

FIGURE 2

Franke's local thin plate splines

In terms of the data considered here, it was for the most part rather nice data, even though some effort was made to include some data with varying densities. Real data exists which is very sparse in certain regions or lies in clumps. Some methods will not work in a reasonable fashion for this type of data, although we have not tried to determine which methods will and which will not. Methods based on quadratic approximations will likely misbehave for such data. In addition, local methods based on distance weighting may have holes in the domain of definition when density varies greatly or when data appears in clumps. Some additional work is necessary to see if there are suitable local methods for such data.

Acknowledgements. This investigation has consumed a great deal of time and effort. Thanks are due to numerous colleagues, among them Greg Nielson, Bob Barnhill, Frank Little, Tom Foley, Rosemary Chang, and others with whom I 
discussed many ideas and who made valuable suggestions (which were sometimes followed!). Thanks are also due to those who supplied working programs, among them Greg Nielson, Hiroshi Akima, Charles Lawson, Tom Foley, and Frank Little.

Very extensive use was made of the computer facility at the Naval Postgraduate School. More recent computations have been done at the Center for Scientific Computation and Interactive Graphics at Drexel University during the author's sabbatical leave.

The author expresses thanks to the referees for suggestions which improved the paper.

Department of Mathematics

Naval Postgraduate School

Monterey, California 93940

1. Hiroshi Akma, "Comments on 'Optimal contour mapping using universal kriging' by Ricardo A. Olea," J. Geophysical Res., v. 80, 1975, pp. 832-836 (with reply).

2. Hiroshi AkIMA, "A method of bivariate interpolation and smooth surface fitting for irregularly distributed data points," ACM Trans. Math. Software, v. 4, 1978, pp. 148-159.

3. HIRoshI AKImA, "Algorithm 526: Bivariate interpolation and smooth fitting for irregularly distributed data points," ACM Trans. Math. Software, v. 4, 1978, pp. 160-164.

4. R. E. BARNHILL, "Representation and approximation of surfaces," in Mathematical Software III (J. R. Rice, Ed.), Academic Press, New York, 1977, pp. 69-120.

5. R. E. BARNHIIL, R. P. DUBE \& F. F. LITTLE, Shepard's Surface Interpolation Formula: Properties and Extensions, CAGD report, University of Utah, 1980.

6. IAN C. BRIGGS, "Machine contouring using minimum curvature," Geophysics, v. 39, 1974, pp. 39-48.

7. Jim Brown, Peter Dube \& Frank Little, Smooth Interpolation with Vertex Functions (manuscript).

8. I. K. Crain \& B. K. Bhattacharyya, "Treatment of nonequispaced two dimensional data with a digital computer," Geoexploration, v. 5, 1967, pp. 173-194.

9. Jean Duchon, Fonctions-Spline du Type Plaque Mince en Dimencion 2, Report \#231, Univ. of Grenoble, 1975.

10. Jean Duchon, Fonctions-Spline à Energie Invariate par Rotation, Report \#27, Univ. of Grenoble, 1976.

11. Jean Duchon, "Interpolation des fonctions de deux variables suivant le principe de la flexion des plaques minces," R.A.I.R.O. Anal. Numér., v. 10, 1976, pp. 5-12.

12. JeAn DuChon, "Splines minimizing rotation invariant semi-norms in Sobolev spaces," in Constructive Theory of Functions of Several Variables (W. Schempp and K. Zeller, Eds.), Lecture Notes in Math. Vol. 571, Springer-Verlag, Berlin and New York, 1977, pp. 85-100.

13. JAmes C. Ferguson, "Multivariable curve interpolation," J. Assoc. Comput. Mach., v. 11, 1964, pp. 221-228.

14. Thomas Alfred Foley, JR., Smooth Multivariate Interpolation to Scattered Data, Ph. D. Dissertation, Arizona State University, 1979.

15. Thomas A. Foley \& Gregory M. Nielson, "Multivariate interpolation to scattered data using delta iteration," in Approximation Theory III (E. W. Cheney, Ed.), Academic Press, New York, 1980, pp. 419-424.

16. Richard Franke, "Locally determined smooth interpolation at irregularly spaced points in several variables," J. Inst. Math. Appl., v. 19, 1977, pp. 471-482.

17. RICHARD FranKE, Smooth Surface Approximation by a Local Method of Interpolation at Scattered Points, Naval Postgraduate School, NPS-53-78-002, 1978.

18. Richard Franke, A Critical Comparison of Some Methods for Interpolation of Scattered Data, Naval Postgraduate School, TR \#NPS-53-79-003, 1979. (Available from NTIS, \# AD-A081 688/4.)

19. Richard FrANKe \& GREGORY NiELSON, "Smooth interpolation of large sets of scattered data," Internat. J. Numer. Methods Engrg., v. 15, 1980, pp. 1691-1704.

20. C. M. Gold, J. D. Charters \& J. RAMSDEN, "Automated contour mapping using triangular element data structures and an interpolant over each irregular triangular domain," Comput. Graphics, v. 11, 1977, pp. 170-175. 
21. William J. Gordon \& James A. Wixom, "Shepard's method of "metric interpolation" to bivariate and multivariate interpolation," Math. Comp., v. 32, 1978, pp. 253-264.

22. R. L. HaRder \& R. N. Desmarais, "Interpolation using surface splines," J. Aircraft, v. 9, 1972, pp. 189-191.

23. Rolland L. HARDY, "Multiquadric equations of topography and other irregular surfaces," $J$. Geophys. Res., v. 76, 1971, pp. 1905-1915.

24. Rolland L. HARDY, “Analytical topographic surfaces by spatial intersection," Photogrammetric Engineering, v. 38, 1972, pp. 452-458.

25. ROLLAND L. HARDY, "Research results in the application of multiquadric equations to surveying and mapping problems," Surveying and Mapping, v. 35, 1975, pp. 321-332.

26. RollaNd L. HARDY, Geodetic Applications of Multiquadric Equations, Iowa State Univ. TR \# 76245 (NTIS PB 255296), 1976.

27. Rolland L. HARDy, “Least squares prediction,” Photogrammetric Eng. and Remote Sensing, v. 43, 1977, pp. 475-492.

28. Rolland L. Hardy, The Application of Multiquadric Equations and Point Mass Anomaly Models to Crustal Movement Studies, NOAA TR NOS 76, NGS 11, 1978.

29. Rolland L. HARDY \& W. M. GopFERT, "Least squares prediction of gravity anomalies, geoidal undulations, and deflections of the vertical with multiquadric harmonic functions," Geophys. Res. Letters, v. 10, 1975, pp. 423-426.

30. J. R. Jancattus \& J. L. JunkIns, “Modeling irregular surfaces,” Photogrammetric Eng. and Remote Sensing, v. 39, 1973, pp. 413-420.

31. J. R. Jancartus \& J. L. JunkINS, "Modeling in $n$ dimensions using a weighting function approach,” J. Geophys. Res., v. 79, 1974, pp. 3361-3366.

32. J. L. JUNKINS, G. W. MilleR \& J. R. JANCAITUS, “A weighting function approach to modeling of irregular surfaces,” J. Geophys. Res., v. 78, 1973, pp. 1794-1803.

33. P. LANCASTER, "Moving weighted least-squares methods," in Polynomial and Spline Approximation (B. N. Sahney, Ed.), Reidel, Dordrecht, 1979, pp. 103-120.

34. P. LanCaster \& K. Salkauskas, Surfaces Generated by Moving Least Squares Methods, Research Paper No. 438, Dept. of Math. and Stat., The Univ. of Calgary, Calgary, Alberta, Canada, 1979. .

35. C. L. LAwson, "Software for $C^{1}$ surface interpolation," in Software III (J. R. Rice, Ed.), Academic Press, New York, 1977, pp. 159-192.

36. Frank LiTtLe, CAGD report, University of Utah. (Forthcoming.)

37. A. Marechal \& J. Serra, "Random kriging," in Geostatistics (Daniel F. Merriam, Ed.), Plenum Press, New York, 1970, pp. 91-112.

38. G. MAtheron, "Random functions and their applications in geology," in Geostatistics (Daniel F. Merriam, Ed.), Plenum Press, New York, 1970, pp. 79-87.

39. G. Matheron, "The intrinsic random functions and their applications," Adv. in Appl. Probab., v. 5,1973 , pp. $439-468$.

40. A. D. MAUDE, "Interpolation-Mainly for graph plotters," Comput. J., v. 16, 1973, pp. 64-65.

41. DeRmot H. MCLaIN, "Drawing contours from arbitrary data points," Comput. J., v. 17, 1974, pp. 318-324.

42. DeRmot H. MCLAIN, “Two dimensional interpolation from random data," Comput. J., v. 19, 1976, pp. 178-181; also errata, ibid., v. 19, 1976, p. 384.

43. Jean Mennguet, "Multivariate interpolation at arbitrary points made simple," $Z$. Angew. Math. Phys., v. 30, 1979, pp. 292-304.

44. Jean MeINGUet, "An intrinsic approach to multivariate spline interpolation at arbitrary points," in Polynomial and Spline Approximation (B. N. Sahney, Ed.), Reidel, Dordrecht, 1979, pp. 163-190.

45. G. M. Nielson, “Minimum norm interpolation in triangles," SIAM J. Numer. Anal., v. 17, 1980, pp. 44-62.

46. Gregory M. Nielson, A Method for Interpolating Scattered Data Based Upon a Minimum Network. (Manuscript.)

47. Ricardo O. Olea, “Optimal contour mapping using universal kriging," J. Geophys. Res., v. 79, 1974, pp. 695-702.

48. Chester R. Pelto, Thomas A. Elkins \& H. A. Boyd, "Automatic contouring of irregularly spaced data," Geophysics, v. 33, 1968, pp. 424-430.

49. M. J. D. Powell \& M. A. SABIN, "Piecewise quadratic approximation on triangles," ACM Trans. Math. Software, v. 3, 1977, pp. 316-325.

50. JeAN-Michel Rendu, "Disjunctive kriging: Comparison of theory with actual results," Math. Geol., v. 12, 1980, pp. 305-320. 
51. M. A. SABIN, "Contouring-A review of methods for scattered data," in Mathematical Methods in Computer Graphics and Design (K. W. Brodlie, Ed.), Academic Press, New York, 1980, pp. 63-85.

52. L. L. SCHUMAKRR, "Fitting surfaces to scattered data," in Approximation Theory II (G. G. Lorentz, C. K. Chui \& L. L. Schumaker, Eds.), Academic Press, New York, 1976, pp. 203-268.

53. Donald ShePARD, A Two-Dimensional Interpolation Function for Irregularly Spaced Data, Proc. 23rd Nat. Conf. ACM, 1968, pp. 517-523.

54. W. L. VirtrTow, Interpolation to Arbitrarily Spaced Data, Ph. D. Dissertation, Dept. of Math., Univ. of Utah, 1978.

55. User Manual for "Surface Gridding Library", Dynamic Graphics, 2150 Shattuck Avenue, Berkeley, Calif., 1978. 